\title{
Delay Analysis for TDMA Schemes with Packet Recombining
}

\author{
Miguel Pereira ${ }^{1,2,3}$, Luís Bernardo ${ }^{1,3}$, Rui Dinis ${ }^{1,2}$, Rodolfo Oliveira ${ }^{1,3}$, \\ Paulo Carvalho ${ }^{1,3}$, and Paulo Pinto ${ }^{1,3}$ \\ ${ }^{1}$ FCT-UNL, Universidade Nova de Lisboa, Portugal \\ ${ }^{2}$ IT, Instituto de Telecomunicações, Portugal \\ ${ }^{3}$ UNINOVA, Monte da Caparica, Portugal \\ \{miguelpereira, $1 \mathrm{fl} \mathrm{b}, \mathrm{rdinis}, \mathrm{rado}$ @ fct.unl.pt, \\ pmc@uninova.pt, pfp@fct.unl.pt
}

\begin{abstract}
This paper considers the use of SC modulations (Single-Carrier) with FDE (Frequency-Domain Equalization) with low-complexity soft combining ARQ schemes (Automatic Repeat reQuest). With our technique, packets associated to different transmission attempts are combined in a soft way, allowing improved performances. Its low complexity makes it particularly interesting for the uplink of wireless systems. This paper proposes an accurate analytical model for a TDMA (Time Division Multiple Access) scheme where packet combining ARQ is applied. It evaluates the uplink non-saturated packet delay for a generic message arrival process. Our analytical results are validated using physical and MAC layer simulations.
\end{abstract}

Keywords: Delay analysis, ARQ schemes, Mac protocol and Markov Chains.

\section{Introduction}

Packet errors can be significant in wireless systems due to fading and shadowing effects, which may lead to significant decrease in the receiving power. The traditional approach to cope with an erroneous packet is to discard it, and ask for its retransmission, which corresponds to the conventional ARQ techniques (Automatic Repeat reQuest). The major problem with conventional ARQ techniques is that persistent unfavorable propagation conditions may originate a very high PER (Packet Error Rate). The individual packet error probability is not affected by the number of packet retransmissions because the information contained in signal of the discarded packets is not used. Hybrid ARQ/FEC (Forward Error Correction) strategies were proposed $[1,2]$ to cope with these scenarios. They retain the signal associated to an erroneous packet and they may ask for additional redundancy. The traditional H-ARQ (Hybrid $\mathrm{ARQ} / \mathrm{FEC}$ ) approach relies on a powerful error correcting code that is punctured, to increase the data rate. Successive retransmissions reduce the puncturing, and therefore increase the error correction capacities of the code. This limits the error correction capability to the base code capabilities. We proposed on [3] the use of soft packet combining techniques to implement H-ARQ on SC modulations (Single Carrier) with FDE (Frequency-Domain Equalization). It is based on repetition codes with soft decision, which is not bounded by the performance of the basic code. The use of H-ARQ 
techniques improve the network throughput compared to conventional ARQ technique because the packet error probability after retransmission $i\left(p_{i}\right)$ is usually lower than $p_{i-1}$. It also reduces the average packet delay.

In this paper we study the performance of H-ARQ techniques for SC-FDE schemes. SC-FDE schemes are generally accepted as one of the best candidates for the uplink of future broadband wireless systems [4]. We consider a TDMA (Time Division Multiple Access) scheme, where a station shares a multiple access communications channel by transmitting its messages during its dedicated time slots, and a generic packet arrival distribution function.

TDMA is used in several current wireless network systems $(802.16,802.11$ s, etc.) when hard QoS (Quality of Service) guarantees are needed. TDMA was the focus of an extensive list of past works (e.g. [5, 6, 7]), which assumed a constant packet error probability for the different retransmissions. ARQ error control was analyzed in [5] for a Poisson distributed message arrival process. Rubin and Zhang [6] analyzed the message delay for TDMA using multiple contiguous-slot assignments, considering a generic message arrival process. Chen and Chang [7] proposed a delay analysis model for TDMA model where slot assignment is characterized by a random variable, for a Poisson distributed message arrival process.

\section{Contribution to Technological Innovation}

This paper is the first to propose an exact analytical model concerning the queue-size and the message delay analysis of the TDMA system with an H-ARQ technique, for a generic message arrival distribution. This model is validated for Poisson traffic using ns-2, showing a significant packet delay reduction compared to the conventional ARQ technique.

\section{System Overview}

In this paper we consider the uplink transmission in an SC-FDE system. The timedomain block associated to a given user (i.e., the corresponding packet) is $\left\{a_{n} ; n=0,1, \ldots, N-1\right\}$, where $a_{n}$ is selected from a given constellation and $N$ is the DFT size (Discrete Fourier Transform). As with other block transmission techniques, a suitable cyclic prefix is added to each time-domain block.

If we detect errors in the packet we ask for its retransmission, but we store the signal associated to each transmission attempt. Although we could keep trying to transmit the packet until there were no errors, in practice there is a maximum number of transmission attempts $N_{R}$. If we fail after $N_{R}$ attempts we need to change the transmission parameters (transmit power, carrier frequency, base station, etc.) since the channel is too bad.

The packet associated to $r$ th attempt to transmit $\left\{a_{n} ; n=0,1, \ldots, N-1\right\}$ is $\left\{a_{n}^{(r)} ; n=0,1, \ldots, N-1\right\}$. Naturally, $a_{n}^{(1)}=a_{n}$; in the following it will be clear that we can use $a_{n}^{(r)} \neq a_{n}$ for $r>1$ to improve the performance in the presence of strong inband interference. 
The received signal associated to the $r$ th transmission attempt is sampled and the cyclic prefix is removed, leading to the time-domain block $\left\{y_{n}^{(r)} ; n=0,1, \ldots, N-1\right\}$. If the cyclic prefix is longer than the overall channel impulse response then the corresponding frequency-domain block is $\left\{Y_{k}^{(r)} ; k=0,1, \ldots, N-1\right\}$, where

$$
Y_{k}^{(r)}=A_{k}^{(r)} H_{k}^{(r)}+N_{k}^{(r)}+I_{k}^{(r)},
$$

with $N_{k}^{(r)}$ denoting the channel noise and $I_{k}^{(r)}$ the corresponding interference. $\left\{A_{k}^{(r)} ; k=0,1, \ldots, N-1\right\}$ is the DFT of $\left\{a_{n}^{(r)} ; n=0,1, \ldots, N-1\right\}$ and $H_{k}^{(r)}$ is the overall channel frequency response for the $r$ th transmission attempt.

Let us assume that we have $R$ versions of the packet (i.e., there were $R$ transmission attempts). Our receiver, which is based on the IB-DFE receivers proposed in $[8,9]$, is depicted in fig 1 . We have an iterative frequency-domain receiver where, for a given iteration $i$, the frequency-domain samples at the output are given by

$$
\tilde{A}_{k}^{(i)}=\sum_{r=1}^{R} F_{k}^{(r, i)} Y_{k}^{(r)}-B_{k}^{(i)} \bar{A}_{k}^{(i-1)}
$$

where $\quad\left\{F_{k}^{(r, i)} ; k=0,1, \ldots, N-1\right\} \quad(r=1,2, \ldots, R) \quad$ and $\quad\left\{B_{k}^{(i)} ; k=0,1, \ldots, N-1\right\} \quad$ can be regarded as the feedforward and the feedback coefficients, respectively. $\left\{\bar{A}_{k}^{(i-1)} ; k=0,1, \ldots, N-1\right\}$ denotes the DFT of the average data estimates $\left\{\bar{a}_{n}^{(i-1)} ; n=0,1, \ldots, N-1\right\}$, where $\bar{a}_{n}$ denotes the average symbol values conditioned to the FDE output, which can be obtain as described in [10].

The optimum feedforward coefficients for a given iteration, can be written as (see [3]), $F_{k}^{(r, i)}=\breve{F}_{k}^{(r, i)} / \gamma^{(i)}$, with $\gamma^{(i)}=\frac{1}{N} \sum_{k=0}^{N-1} \sum_{r=1}^{R} \breve{F}_{k}^{(r, i)} H_{k}^{(r)}$ and

$$
\breve{F}_{k}^{(r, i)}=\frac{H_{k}^{(r)^{*}}}{\alpha+\left(1-\left(\rho^{(i-1)}\right)^{2}\right) \sum_{r^{\prime}=1}^{R}\left|H_{k}^{\left(r^{\prime}\right)}\right|^{2}}, r=1,2, \ldots, R,
$$

where $\alpha=\frac{E\left[\left|N_{k}^{(r)}\right|^{2}\right]}{E\left[\left|A_{k}\right|^{2}\right]}$ (i.e., $\alpha$ is the inverse of the SNR (Signal-to-Noise Ratio)) and the correlation coefficient $\rho^{(i)}$ is given by

$$
\rho^{(i)}=\frac{1}{2 N} \sum_{n=0}^{N-1}\left(\left|\operatorname{Re}\left\{\bar{a}_{n}^{(i)}\right\}\right|+\left|\operatorname{Im}\left\{\bar{a}_{n}^{(i)}\right\}\right|\right) .
$$

The optimum feedback coefficients (also to minimize the signal-to-noise plus interference ratio) are given by

$$
B_{k}^{(i)}=\sum_{r^{\prime}=1}^{R} F_{k}^{\left(r^{\prime}, i\right)} H_{k}^{\left(r^{\prime}\right)}-1
$$




\section{The System Model}

In this section we present the exact message delay and queue-size analysis for the system.

\subsection{System Characterization}

We consider a communication channel on a TDMA basis. Time is divided into equal length slots, each of duration equal to 1 block data of 32 bytes. The start of a message transmission across the channel must coincide with the beginning of a slot. The success of an arriving message is given by $q_{l}, 0<q_{l}<1$, with $l, 1 \leq l \leq R$, representing the transmission number of each message and $R$ the maximum number of retransmissions allowed for each message. Each packet contains 32 bytes, so that a packet transmission time is equal to 1 slot. $N_{n}$ represents the number of messages arrivals at the station during the $n$th slot, according to a stochastic process. We assume that $\left\{N_{n}, n \geq 1\right\}$ is a sequence of i.i.d. random variables, and we set $a_{m}=P\left(N_{n}=m\right), m \geq 0$, and $\alpha=E\left[N_{n}\right]$ where $\alpha$ is the mean number of messages arrivals per slot. The station is allocated a possible multiple contiguous slots per frame $N$, with $N \geq 1$. A frame contains $N+L$ slots, with $L \geq 1$, representing the group of slots allocated to other stations. Messages can only be transmitted in those $N$ dedicated slots during a frame. This section proposes a complete solution for the delay with $N=1$, and characterizes the system dynamics for a generic $N$ value.

\subsection{The Generating Function of the Queue Size}

Let $X_{k}$ represent the number of messages in the station's queue at the start of slot $k$ (including message arrivals that will occur during slot $k-1$ ), and let $Z_{k}$ represent the number of transmissions of one message at the same slot. So, at the start of slot $k$ we have $X_{k}$ messages in queue, and we will transmit the corresponding message for the $Z_{k}$ th time. The process $\left\{\left(X_{k}, Z_{k}\right) k \geq 1\right\}$ is not Markovian because it is time dependent. To make it Markovian we use one supplementary variable, $Y_{k}$, to represent the slot index in the frame, $1 \leq Y_{k} \leq N+L$. With this we define an embedded Markov Chain $\left\{\left(X_{k}, Y_{k}, Z_{k}\right), k \geq 1\right\}$.

It can be shown that the system is stable if the average number of messages arrivals per slot $\alpha$ is less than the average number of messages transmitted per slot. Under the stability condition, the conditional steady-state distribution $\left\{X_{k}\right\}$ given that $Y_{k}=j$ and $Z_{k}=l$ and the steady-state distribution of $\left\{X_{k}\right\}$ in the Cesaro sense exists. Denote

$$
P_{j, i, l}=\lim _{k \rightarrow \infty} P\left(X_{k}=i \mid Y_{k}=j, Z_{k}=l\right)
$$




$$
\pi(i)=\lim _{J \rightarrow \infty} \frac{1}{J} \sum_{k=1}^{J} \sum_{j=1}^{N+L} \sum_{l=1}^{R} P\left(X_{k}=i \mid Y_{k}=j, Z_{k}=l\right)
$$

Next, we define a set of $z$-transforms, $|z| \leq 1$.

$$
\begin{gathered}
U_{j, l}(z)=\sum_{i=0}^{\infty} P_{j, i, l} z^{i} \quad 1 \leq j \leq N+L, 1 \leq l \leq R \\
\Pi(z)=\sum_{i=0}^{\infty} \pi(i) z^{i} \\
A(z)=\sum_{i=0}^{\infty} a_{i} z^{i} A^{\prime}(1)=\alpha, A^{\prime \prime}(1)=\alpha_{2}
\end{gathered}
$$

The steady-state probabilities referred previously satisfy the following equilibrium equations. For $i \geq 0$

$$
\begin{gathered}
P_{1, i, l}=\sum_{m=0}^{i} a_{m} P_{N+L, i-m, l} \\
P_{j, i, l}=\sum_{m=0}^{i} a_{m} P_{j-1, i-m, l}, \quad N+2 \leq j \leq N+L \\
P_{j, i, 1}=a_{i} P_{j-1,0,1}+\sum_{l=1}^{R-1} q_{l} \sum_{m=0}^{i} a_{m} P_{j-1, i+1-m, l}+\sum_{m=0}^{i} a_{m} P_{j-1, i+1-m, R}, \quad 2 \leq j \leq N+1 \\
P_{j, i, l}=\left(1-q_{l-1}\right) \sum_{m=0}^{i-1} a_{m} P_{j-1, i-m, l-1}, \quad 2 \leq l \leq R, 2 \leq j \leq N+1
\end{gathered}
$$

Looking the fact that $P\left(Y_{k}=j\right)=(N+L)^{-1}$, for $1 \leq j \leq N+L$, we have

$$
\pi(i)=\frac{1}{N+L} \sum_{j=1}^{N+L} \sum_{l=1}^{R} P_{j, i, l}
$$

Considering (8) and (9) we then obtain

$$
\Pi(z)=\frac{1}{N+L} \sum_{j=1}^{N+L} \sum_{l=1}^{R} U_{j, l}
$$

The objective is to derive an expression for $\Pi(z)$. Multiplying the $i$ th equation of (11) to (14) by $z^{i}$ and summing over $i$, we obtain the following set of equations

$$
\begin{aligned}
& U_{1, l}(z)=A(z) U_{N+L, l}(z) \\
& U_{j, l}(z)=A(z) U_{j-1, l}(z), \quad N+2 \leq j \leq N+L \\
& U_{j, l}(z)=\left(1-q_{l-1}\right) A(z)\left(U_{j-1, l-1}(z)-P_{j-1,0, l-1}\right), \quad 2 \leq j \leq N+1
\end{aligned}
$$




$$
\begin{array}{r}
U_{j, 1}(z)=A(z) P_{j-1,0,1}+\sum_{l=1}^{R-1} q_{l} A(z) z^{-1}\left(U_{j-1, l}(z)-P_{j-1,0, l}\right)+A(z) z^{-1} \times\left(U_{j-1, R}(z)-P_{j-1,0, R}\right) . \\
2 \leq j \leq N+1
\end{array}
$$

Henceforth this paper considers only the resolution of $\left\{X_{k}\right\}$ 's steady-state distribution for $N=1$. Using (17) to (20) iteratively, and due to the fact of $P_{1,0, l}=0$ for $l \geq 2$ we obtain after some algebric manipulations

$$
U_{2,1}(z)=\frac{f_{0}(z)}{g_{0}(z)}
$$

where

$$
\begin{gathered}
f_{0}(z)=A(z) P_{1,0,1}\left[1-z^{-1}\left(q_{1}+Q_{R} A(z)^{(R-1)(L+1)}+\sum_{l=2}^{R-1} q_{l} Q_{l} A(z)^{(l-1)(L+1)}\right)\right] \\
g_{0}(z)=1-z^{-1}\left(q_{1} A(z)^{L+1}+Q_{R} A(z)^{R(L+1)}+\sum_{l=2}^{R-1} q_{l} Q_{l} A(z)^{l(L+1)}\right)
\end{gathered}
$$

and

$$
Q_{X}=\prod_{m=1}^{X-1}\left(1-q_{m}\right)
$$

By the normalization condition, $\sum_{l=1}^{R} U_{2, l}(1)=1$, we then obtain,

$$
P_{1,0,1}=\left[\left(1+\sum_{l=2}^{R} Q_{l}\right) \frac{D_{z}}{L_{z}}-\sum_{l=2}^{R} Q_{l}\right]^{-1}
$$

where $U_{2,1}(1)$ is determined applying the L'Hôspital rule and is equal to

$$
U_{2,1}(1)=\frac{f_{1}}{g_{1}}
$$

and where

$$
\begin{gathered}
f_{1}=P_{1,0,1} D_{z} \\
D_{z}=1+\alpha\left(1-q_{1}-Q_{R}[(R-1)(L+1)+1]-\sum_{l=2}^{R-1} q_{l} Q_{l}[(l-1)(L+1)+1]\right) \\
g_{1}=1-(L+1) \alpha\left[q_{1}+Q_{R} R+\sum_{l=2}^{R-1} q_{l} Q_{l} l\right]
\end{gathered}
$$


From (16) we obtain the generating function

$$
\Pi(z)=\frac{1}{L+1}\left(\sum_{j=0}^{L} A(z)^{j}\right)\left[U_{2,1}(z)+\sum_{l=2}^{R} P(z)\right]
$$

where

$$
P(z)=\left(Q_{l} A(z)^{(l-2)(L+1)+1}\right)\left(A(z)^{L} U_{2,1}(z)-P_{1,0,1}\right)
$$

\subsection{The Mean Queue Size}

The mean the number of messages in the station's queue can be computed by taking the derivative of $\Pi(z)$ and letting z equal to 1 . From (30) we obtain

$$
\begin{aligned}
\Pi^{\prime}(1)= & \frac{\sum_{j=0}^{L} j \alpha}{L+1}\left[U_{2,1}(1)+\sum_{l=2}^{R} Q_{l}\left(U_{2,1}(1)-P_{1,0,1}\right)\right]+ \\
& {\left[U_{2,1}^{\prime}(1)+\sum_{l=2}^{R} Q_{l}\left[(l-1)(L+1) \alpha U_{2,1}(1)+U_{2,1}^{\prime}(1)-[(l-2)(L+1)+1] \alpha P_{1,0,1}\right]\right] }
\end{aligned}
$$

To compute $U_{2,1}^{\prime}(1)$ we apply twice the L'Hôpital's rule to obtain

$$
U_{2,1}^{\prime}(1)=\frac{f_{2} g_{1}-f_{1} g_{2}}{2 g_{1}^{2}}
$$

where

$$
\begin{aligned}
f_{2}= & P_{1,0,1}\left[2 \alpha+\alpha_{2}\left(1-q_{1}\right)-Q_{R}[(R-1) \times(L+1)+1]\left[(R-1)(L+1) \alpha^{2}+\alpha_{2}\right]-\right. \\
& \left.\sum_{l=2}^{R-1} q_{l} Q_{l}[(l-1)(L+1)+1]\left[(l-1)(L+1) \alpha^{2}+\alpha_{2}\right]\right] \\
& g_{2}=-q_{1}(L+1)\left(L \alpha^{2}+\alpha_{2}\right)-Q_{R} R(L+1)\left([R(L+1)-1] \alpha^{2}+\alpha_{2}\right)- \\
& \sum_{l=2}^{R-1} q_{l} Q_{l} l(L+1)\left[(l(L+1)-1) \alpha^{2}-\alpha_{2}\right]
\end{aligned}
$$

\subsection{Delay Analysis}

The message delay $E[D]$ is defined as the time interval elapsed between the message arrival and departure times. It can be calculated as the sum of the time elapsed between the message arrival and the beginning of the next slot (equal to one half-slot) plus the number of slots elapsed between the slot of its arrival and the instant it is 
fully transmitted from the system. For the general batch arrival case, this last component of the delay can be obtained using Little's formula assuming a first-come-firstserved (FCFS) service discipline. That is,

$$
E[D]=\frac{E[X]}{\alpha}+\frac{1}{2}
$$

where $E[X]=\Pi^{\prime}(1)$ is given by (33). This equation can be used with any message arrival distribution, as long as the message arrival process, $A(Z)$, is defined.

The next section validates the proposed model, assuming arrivals to be governed by a Poisson process, so

$$
A(z)=e^{-\lambda(1-z)}, A^{\prime}(1)=\alpha=\lambda .
$$

\section{Simulations}

In this section, we present a set of performance results concerning the proposed packet combining ARQ scheme for a TDMA uplink channel. Each FFT (Fast Fourier Transform) block has $\mathrm{N}=256$ data symbols and are selected from a QPSK constellation, with Gray mapping totalling 512 bits per data block. The data packets correspond to one FFT block, with 32 bytes. We consider an uncoded scenario where the channels for each packet retransmission remains fixed. To allow an efficient packet separation we consider the cyclic-shifted versions of the FFT-blocks for each retransmission attempt which formally is equivalent to have cyclic-shifted versions of the channel in each retransmission attempt [3].

We assumed perfect synchronization and channel estimation conditions. We consider, as referred earlier, one slot per station $(N=1)$ and 8 stations $(L=8)$ generating traffic following a Poisson process with $\lambda$ packets/slot.

Fig. 1 shows the average PER of our interactive receiver when we have $l$ transmission attempts $\left(q_{l}\right)$. It shows a significant PER reduction with a $l$ 's increment, especially when the number of $R$ is not too large (the $E_{b} / N_{0}$ gains for PER $=0.5$ are about $4 \mathrm{~dB}$ when $l=1,2 \mathrm{~dB}$ when $l=2$ and $1 \mathrm{~dB}$ when $l=3$ ). This is not surprising, since the total transmitted power grows with $l$ and we take full advantage of all transmitted power.

We implemented the proposed packet combining approach in the ns- 2 simulator [12]. We assumed that $q_{l}$ for packets is a mean-ergodic process, and we modeled the PER variation with $l$ using the average PER values measured in the first set of experiments, and represented in Fig. 1. The average $q_{l}$ in function of $E_{b} / N_{0}$ is introduced as a matrix parameter for the simulations.

Fig. 2 depicts the simulation and the analytical model message delay in function of $E_{b} / N_{0}$ for different Poisson traffic load (when $1 / \lambda$ equal to 10, 20 and 30 packets), for $R=4$ (it attempts up to 4 transmissions per packet). The analytical results were computed using 43 and 47 . It shows that the measured delay follows the analytical 
model values, thus validating it. It also shows that message delay decreases as $E_{b} / N_{0}$ increases. Notice that packet combining allows a finite delay even for a initial very high PER $\left(q_{1} \approx 0\right.$ for $3 \mathrm{~dB}$ ), clearly showing its advantage compared to a classical TDMA scheme.

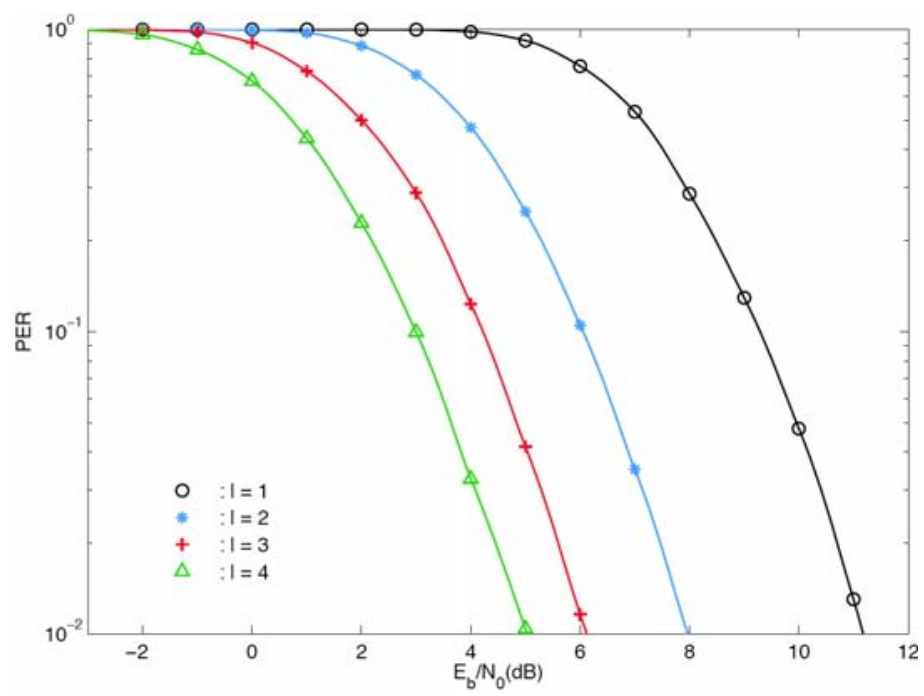

Fig. 1. PER performance for a single packet transmission

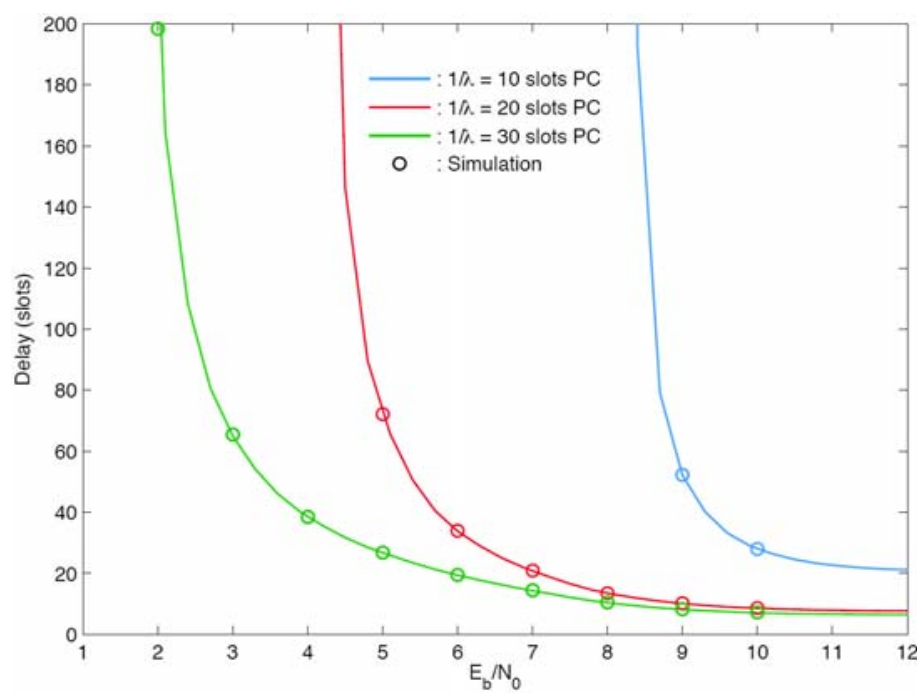

Fig. 2. Message delay with $E_{b} / N_{0}$ for Poisson traffic and $R=4$ 


\section{Conclusions}

In this paper, we have carried out exact message delay and queue-size analysis for a single slot per frame packet-switched TDMA applying a packet combining ARQ scheme. We propose a generic analytical model that applies to any message arrival distribution. The proposed analysis was validated using simulations for a SC-FDE scheme, showing its correctness. The results show the significant gains that can be obtained using a packet combining ARQ scheme compared to a classical TDMA scheme.

Future work includes the testing of the model with other message arrival processes, including long-tail processes. At model level extended models are required to handle multiple slots per frame, and other queueing disciplines.

\section{References}

1. Hagenauer, J.: Rate-compatible Punctured Convolutional Codes (RPCP Codes) and Their Applications. IEEE Trans. on Communications 36(4), 389-400 (1988)

2. Gusmão, A., Dinis, R., Esteves, N.: Adaptive HARQ Schemes Using Punctured RR Codes for ATM-compatible Broadband Wireless Communications. In: IEEE VTC 2009 (Fall), Amsterdam (1999)

3. Dinis, R., Carvalho, P., Martins, J.: Soft Combining ARQ Techniques for Wireless Systems Employing SC-FDE Schemes. In: IEEE ICCCN 2008, St. Thomas U.S. Virgin Islands (2008)

4. Falconer, D., Ariyavisitakul, S., Benyamin-Seeyar, A., Eidson, B.: Frequency Domain Equalization for Single-Carrier Broadband Wireless Communications. IEEE Comm. Mag. 40(4), 58-66 (2002)

5. Saeki, B.H., Rubin, I.: An Analysis of a TDMA Channel Using Stop-and-Wait, Block, and Select-and Repeat ARQ Error Control. IEEE Trans. on Communications 30, 1162-1173 (1982)

6. Rubin, I., Zhang, Z.: Message Delay Analysis for TDMA Schemes Using Continous-Slot Assignments. IEEE Trans. on Communications 40(4), 730-737 (1992)

7. Chen, Y.J., Chang, J.F.: Per connection delay analysis of a frame-based TDMA/CDMA MAC protocol. Performance Evaluation 57, 19-55 (1992)

8. Benvenuto, N., Tomasin, S.: Block Iterative DFE for Single Carrier Modulation. IEE Elec. Let. 39(19), 1144-1145 (2002)

9. Dinis, R., Gusmão, A., Esteves, N.: On Broadband Block Transmission over Strongly Frequency-Selective Fading Channels. In: Proc. Wireless 2003, Calgary (2003)

10. Gusmão, A., Torres, P., Dinis, R., Esteves, N.: A Class of Iterative FDE Techniques for Reduced-CP SC-Based Block Transmission. In: Int. Symposium on Turbo Codes (2006)

11. The Network Simulator ns-2 (2.33), http: / /www. isi.edu/nsnam/ns / 